\title{
Article \\ FGFR1 Amplification and Response to Neoadjuvant Anti-HER2 Treatment in Early HER2-Positive Breast Cancer
}

\author{
María Gaibar ${ }^{1} \oplus$, Apolonia Novillo ${ }^{2} \oplus$, Alicia Romero-Lorca ${ }^{2}$, Diego Malón ${ }^{3}$, Beatriz Antón ${ }^{3}$, Amalia Moreno $^{3}$ \\ and Ana Fernández-Santander $2, * \mathbb{B}$ \\ 1 Health Sciences Faculty, Universidad Francisco de Vitoria, 28223 Madrid, Spain; maria.gaibar@ufv.es \\ 2 Biomedical and Health Sciences Faculty, Universidad Europea de Madrid, 28670 Madrid, Spain; \\ apolonia.novillo@universidadeuropea.es (A.N.); alicia.romero@universidadeuropea.es (A.R.-L.) \\ 3 Department of Oncology, University Hospital of Fuenlabrada, 28942 Madrid, Spain; \\ diego.malon@salud.madrid.org (D.M.); beatriz.anton@salud.madrid.org (B.A.); \\ amorenot@salud.madrid.org (A.M.) \\ * Correspondence: ana.fernandez@universidadeuropea.es; Tel.: +34-912115288
}

Citation: Gaibar, M.; Novillo, A.; Romero-Lorca, A.; Malón, D.; Antón,

B.; Moreno, A.; Fernández-Santander, A. FGFR1 Amplification and Response to Neoadjuvant Anti-HER2 Treatment in Early HER2-Positive Breast Cancer. Pharmaceutics 2022, 14 , 242. https://doi.org/10.3390/ pharmaceutics14020242

Academic Editors: Francisco Abad Santos, Pablo Zubiaur and Pedro Dorado

Received: 22 November 2021 Accepted: 18 January 2022

Published: 20 January 2022

Publisher's Note: MDPI stays neutral with regard to jurisdictional claims in published maps and institutional affiliations.

Copyright: (c) 2022 by the authors. Licensee MDPI, Basel, Switzerland. This article is an open access article distributed under the terms and conditions of the Creative Commons Attribution (CC BY) license (https:// creativecommons.org/licenses/by/ $4.0 /)$.

\begin{abstract}
HER2-positive breast cancer (BC) is an aggressive subtype that affects $20-25 \%$ of $B C$ patients For these patients, neoadjuvant therapy is a good option that targets a pathological complete response (pCR) and more breast-conserving surgery. In effect, the outcomes of patients with HER2-positive BC have dramatically improved since the introduction of anti-HER2 antibodies such as trastuzumab (TZ) and/or pertuzumab (PZ) added to chemotherapy. This study sought to examine whether correlation exists between copy number variations (CNVs) in several genes related to the PI3K/AKT pathway (HER2, FGFR1, PIK3CA, AKT3 and MDM2) and the efficacy of anti-HER2 neoadjuvant treatment in patients with early HER2-positive BC. Forty-nine patients received TZ or PZ/TZ and chemotherapy as neoadjuvant treatment. Gene CNVs were determined by quantitative polymerase chain reaction on paraffin-embedded biopsy specimens. The response to 6 months of therapy was assessed by Miller-Payne grading of the tumor on surgical resection; grades 4 and 5, indicating $>90 \%$ tumor reduction, were defined as a good response. A good response was shown by $64.5 \%$ and a pCR by $31.2 \%$ of patients. When stratified by anti-HER2 antibody received and gene CNV, it was found that patients with FGFR1 gene amplification or those with FGFR1 amplification treated with TZ alone showed a poor response ( $p=0.024$ and $p=0.037$, respectively). In the subset of patients treated with $\mathrm{TZ} / \mathrm{PZ}$ combined, the $\mathrm{pCR}$ rate was significantly lower among those showing FGFR1 amplification $(p=0.021)$. Although based on a small sample size, our findings suggest that patients with FGFR1 amplification might benefit less from anti-HER2 antibody therapy.
\end{abstract}

Keywords: HER2-positive breast cancer; anti-HER2 treatment; FGFR1 gene; CNVs; pathological complete response; Miller-Payne grading

\section{Introduction}

The human epidermal growth factor receptor 2 (HER2 or ERBB2) gene occurs in the $17 q 21$ chromosome region and encodes a transmembrane glycoprotein with tyrosine kinase activity. HER2 is involved in the regulation of cell growth, differentiation, and invasion and it is well known that its overexpression is associated with an aggressive phenotype and poor prognosis in breast cancer (BC) patients [1]. The frequency of this HER2-positive phenotype BC is between 20 and $30 \%$ of all BC [2]. Neoadjuvant therapy is a good option for operable early $B C$ and targets a pathological complete response (pCR) detected upon surgery. Besides improving the chances of operability and offering more breast-conserving surgery, neoadjuvant treatment has been reported to improve disease-free survival and overall survival in patients with early stage HER2-positive BC [3].

Trastuzumab (TZ) is the first humanized anti-HER2 monoclonal antibody that acts against the extracellular domain of the human HER2 protein. This antibody inhibits 
the ligand-independent HER2 and HER3 signaling through the PI3K/AKT pathway (phosphoinositide-3-kinase/Akt serine/threonine kinase) that induces antibody-dependent cellular cytotoxicity [4]. Since the introduction of TZ added to chemotherapy (CT) nearly two decades ago, the outcomes of patients with HER2-positive BC have dramatically improved. In the $\mathrm{NOAH}$ trial, addition of $\mathrm{TZ}$ to the treatment regimen for women with locally advanced BC both increased the pCR rate from $19 \%$ to $38 \%$ and led to a significant improvement in event-free survival [5]. Pertuzumab (PZ) is a monoclonal antibody directed against the dimerization domain of HER2 and also works against the HER2 pathway. Dual HER2 blockade induced by adding PZ to TZ in neoadjuvant CT has been shown to increase the $\mathrm{pCR}$ rate compared with $\mathrm{TZ}$ alone, giving rise to a rate of around 50-70\% [6].

The PI3K/AKT pathway can be activated by different signaling molecules apart from ligand-independent HER2 and HER3 signaling or HER2 dimerization. FGFR1 is a member of the FGFR family that exhibits a highly conserved structure among different receptors of fibroblast growth factors. The interaction between fibroblast growth factors and FGFRs has been related to cell differentiation, proliferation, and survival [7]. FGFR and EGFR signaling may mediate the downstream phosphoinositide-3-kinase/Akt serine/threonine kinase (PI3K/AKT) pathway. Akt mediates the negative control of p53 levels through phosphorylation of MDM2, which is necessary for p53 degradation [8]. The activation of MDM2 by Akt and reduction in p53 levels has also been observed in HER2-overexpressing BC cells, with the consequence of promoting tumor progression [9]. FGFR1 gene amplification was observed in $8-15 \%$ of all cases of BC [10], and results in the aberrant activation of the downstream PI3K/AKT pathway, promoting cell cycle progression and apoptosis inhibition.

Breast cancer is a very heterogeneous disease with many different polymorphisms detected among BC patients. In the past few years, frequent gene copy number variations (CNVs) have been identified, such as those affecting the genes FGFR1, HER1, HER2 or PIK3CA, involved in the PI3K/AKT pathway. In effect, CNVs in a wide array of these genes have been attributed an important role in the variability of clinical outcomes [11].

The response to anti-HER2 neoadjuvant treatments for HER2-positive BC varies widely among patients. For instance, $\sim 50 \%$ of patients were found not to benefit from TZ neoadjuvant treatment [12]. Thus, it is extremely important to distinguish between HER2-positive breast cancer patients who are more likely to respond well to anti-HER2 treatment and those who are not. The aim of this study was to examine the possible association between CNVs in five genes related to the PI3K/AKT pathway, HER2, FGFR1, PIK3CA, AKT3 and MDM2, and the efficacy of anti-HER2 blockade neoadjuvant treatment in patients with early HER2-positive BC.

\section{Materials and Methods}

\subsection{Design and Patients}

Forty-nine female patients diagnosed with HER2+ breast cancer were treated at the Oncology Department of the University Hospital of Fuenlabrada, Madrid, Spain, between 2010 and 2018. In each patient, HER2/ER/PR status was determined as part of the routine diagnostic procedure. Patients received TZ or PZ/TZ combo and CT as neoadjuvant therapy to allow for breast-conserving surgery and a pathological complete response. The therapeutic regimens and targeted therapy used were based on current standard of care in each case: (i) anthracycline-based therapy (epirubicin, cyclophosphamide with weekly paclitaxel), (ii) carboplatin-docetaxel therapy, or (iii) taxane monotherapy (weekly paclitaxel). Patient samples and data were collected by medical oncologists at the Oncology Department, in accordance with the Declaration of Helsinki. The study protocol was approved by the Hospital's Ethics Committee (identification code: APR 18/20, September 2018). Informed consent for the experimental use of biopsies/tumors collected for diagnosis was provided by all patients.

Response to treatment was assessed using the Miller-Payne tumor grading system ( $1=$ no tumor reduction, $2=$ up to $30 \%$ tumor reduction, $3=30-90 \%$ tumor reduction, $4=>90 \%$ tumor reduction (considered close to a pCR), and $5=$ no invasive malignant cells 
identifiable in sections from the tumor site (considered a pCR)). For the present purpose, patients showing Miller-Payne grades 4 and 5 were defined as good responders and those with grades 1 to 3 were classified as poor responders. Tumor response grade was assessed after 6 months of treatment at surgery resection.

Overall survival (OS) was determined based on the time from treatment onset to the date of any-cause death or last follow-up.

\subsection{CNV Selection and Analysis}

The five CNVs examined here were selected on the basis of published evidence of somatic alterations in specific genes identified in HER2-positive breast cancer patients (see for instance [13]). The locations of the studied CNVs in the genes HER2, FGFR1, PIK3CA, $A K T 3$ and MDM2 are provided in Table 1. The five genes selected are those involved in the HER2 pathway with an important role in patients showing HER2 overexpression such as the present participants. HER2 and FGFR1 receptors act upon the PI3K/AKT intracellular signaling pathway, and play a key role in cell cycle regulation [14]. AKT phosphorylates and activates MDM2 protein, an important negative regulator of p53.

Table 1. Details of the assays and genes used in the detection of CNVs.

\begin{tabular}{ccccc}
\hline Gene & Thermo Fischer ID & Target Size (bp) & $\begin{array}{c}\text { Location on GRCh38 } \\
\text { (Cytoband) }\end{array}$ & $\begin{array}{c}\text { Gene Location } \\
\text { Identified }\end{array}$ \\
\hline $\begin{array}{c}\text { FGFR1 } \\
\text { (fibroblast growth factor receptor 1) }\end{array}$ & Hs00770365_cn & 75 & $\begin{array}{c}\text { Chr.8: 38457439 } \\
\text { (8p11.23) }\end{array}$ & within exon 5 \\
\hline $\begin{array}{c}\text { HER2 } \\
\text { (ERRB2 receptor tyrosine kinase 2) }\end{array}$ & Hs00709630_cn & 101 & $\begin{array}{c}\text { Chr.17:39723405 } \\
(17 q 12)\end{array}$ & within exon 22 \\
\hline $\begin{array}{c}\text { MDM2 } \\
\text { (MDM2 proto-oncogene) }\end{array}$ & Hs03082319_cn & 99 & $\begin{array}{c}\text { Chr.12: 68835830 } \\
\text { (12q15) }\end{array}$ & overlapping intron \\
PIK3CA exon 11
\end{tabular}

Total DNA was extracted from $2.5 \mathrm{~mm} 3$ of paraffin-embedded tumor biopsies, as required by the QIAamp ${ }^{\circledR}$ DNA FFPE Tissue Kit (Qiagen, Germantown, MD, USA). Each $10 \mu \mathrm{L}$ reaction mixture contained $5 \mathrm{ng}$ of gDNA as the PCR template, $1 \times$ TaqMan Gene Expression PCR Master Mix $(5 \mu \mathrm{L})$ along with $1 \times$ TaqMan $^{\mathrm{TM}}$ Copy Number Assay (Hs00709630_cn, Hs06659652_cn, Hs05793001_cn, Hs03082319_cn, Hs00770365_cn, Table 1) and $1 \times$ TaqMan $^{\mathrm{TM}}$ Copy Number Reference Assay (RNase $p$ ). Real-time PCR plates were run in an QuantStudio 12K Flex Real-Time PCR System (Applied Biosystems, Thermo Fisher Scientific, Waltham, MA, USA) under standard running conditions $\left(95^{\circ} \mathrm{C}\right.$ for $10 \mathrm{~min}$ and 40 two-step cycles consisting of $95^{\circ} \mathrm{C}$ for $15 \mathrm{~s}$ and $60^{\circ} \mathrm{C}$ for $1 \mathrm{~min}$ ) and analyzed with Copy Caller Software (Applied Biosystems, Thermo Fisher Scientific, Waltham, MA, USA). All reactions were performed in quadruplicate.

\subsection{Statistical Analysis}

Quantitative variables are provided as medians and their interquartile range or means and their standard deviation depending on their distribution (Shapiro-Wilk test for normality). For qualitative variables, absolute and relative frequencies are given as percentages. The relationship between each gene's CNV and tumor response (good and poor response) was assessed using the Mann-Whitney or Student $t$ tests according to the normality of the data. In patients stratified according to the anti-HER2 treatment received, the Chi-square test was used to compare rates of good responders to each treatment. Time-to-event data were analyzed using the Kaplan-Meier method. All calculations were performed using the Program Stata v.14.2 (Stata Corp, LLC, Lakeway, TX, USA). Significance was set at $p \leq 0.05$. 


\section{Results}

\subsection{Patient Characteristics}

Table 2 shows the clinical characteristics of the BC patients enrolled. All of them were women (100\%) of median age 50 years (range 28.4-74.6 years). Tumor locations were $42.9 \%$ right side and $57.1 \%$ left side. Most tumors were ductal, $93.9 \%$ compared to $6.1 \%$ for lobular. Histology grades on diagnosis were $14.3 \%, 53.0 \%$ and $32.7 \%$, for grades 1,2 , and 3 , respectively. All patients were positive for HER2, ER and PR and most had no metastasis on diagnosis (95.9\%). The anti-HER2 drug received was TZ in $65.3 \%$ of the patients and TZ $+\mathrm{PZ}$ combo in the remaining $34.7 \%$. Chemotherapy regimens based on current standard guides were anthracycline-based therapy in $22.5 \%$, carboplatin-docetaxel therapy in $61.2 \%$ and taxane monotherapy in $16.3 \%$ (Table 2).

After six months of therapy with $\mathrm{TZ}$ or $\mathrm{PZ} / \mathrm{TZ}$ and $\mathrm{CT}, 64.5 \%$ of the $\mathrm{BC}$ patients showed a good response as indicated by $>90 \%$ tumor reduction (Miller-Payne grade $4+5$ ). A pathological complete response was observed in $31.2 \%$ of patients (Table 2). For one patient, the Miller-Payne response was unknown. After stratifying patients according to the anti-HER2 treatment received, rates of good responders were similar in the patients treated with TZ (58.1\%) versus TZ/PZ $(76.5 \%)(p=0.202)$. No correlation was detected between response to therapy and the clinical characteristics of the patients.

\subsection{Gene Amplification in Relation to Treatment Effectiveness and Survival}

Of the five genes examined, amplification of the FGFR1 gene was significantly associated with the response shown to the anti-HER2 drugs. Hence, patients showing a poor tumor response (Miller-Payne grades 1-3) had significantly more FGFR1 gene amplification $(p=0.024$, Table 3). More FGFR1 amplification was also associated with a poor response in the subgroup of patients treated with TZ alone $(p=0.037)$. Furthermore, within the TZ/PZ combo group, the $\mathrm{pCR}$ rate was significantly lower in patients showing greater FGFR1 gene amplification $(p=0.021)$.

Table 2. Baseline characteristics of the 49 patients enrolled in this study.

\begin{tabular}{cc}
\hline Patient Characteristics & No. (\%) \\
\hline Median age (years) & 50.0, range 28.4-74.6 \\
\hline Gender & - \\
- Female & $(100)$ \\
- Male & $21(42.9)$ \\
\hline Tumor location & $28(57.1)$ \\
- Right-sided & \\
- Left-sided & $46(93.9)$ \\
\hline Histology type & $3(6.1)$ \\
- Ductal & \\
- Lobular & $7(14.3)$ \\
\hline Histology grade & $26(53.0)$ \\
- Grade 1 & $16(32.7)$ \\
- Grade 2 & \\
- Grade 3 & $49(100)$ \\
\hline HER2 status & - \\
- Positive & \\
- Negative & $49(100)$ \\
\hline ER status & - \\
\hline - Positive &
\end{tabular}


Table 2. Cont.

\begin{tabular}{cc}
\hline Patient Characteristics & No. (\%) \\
\hline PR status & $49(100)$ \\
- Positive & - \\
- Negative & $47(95.9)$ \\
Metastasis & $2(4.1)$ \\
- No & $1(2.1)$ \\
- Yes & $7(14.6)$ \\
-1 & $9(18.8)$ \\
-2 & $16(33.3)$ \\
-3 & $15(31.2)$ \\
\hline Miller-Payne response grade & $32(65.3)$ \\
\hline Anti-HER2 drug & $17(34.7)$ \\
\hline - Trastuzumab & $11(22.5)$ \\
\hline - Trastuzumab + pertuzumab & $30(61.2)$ \\
\hline - Carboplatin-docetaxel therapy & $8(16.3)$ \\
\hline
\end{tabular}

Table 3. Association between gene CNVs and anti-HER2 treatment response (Miller Payne 1-3 = poor response, Miller Payne 4-5 = good response).

\begin{tabular}{|c|c|c|c|c|c|c|c|}
\hline Gene & Response & Mean & SD & Median & IQR & Range & $p$ Value \\
\hline \multirow{2}{*}{ HER2 } & $\begin{array}{c}\text { Poor } \\
\mathrm{n}=17\end{array}$ & 3.72 & 5.00 & 2.00 & 2.42 & $0.78-21.63$ & \multirow{2}{*}{0.464} \\
\hline & $\begin{array}{l}\text { Good } \\
\mathrm{n}=31\end{array}$ & 3.75 & 3.14 & 3.03 & 3.82 & $0.27-12.19$ & \\
\hline \multirow{2}{*}{ FGFR1 } & $\begin{array}{c}\text { Poor } \\
\mathrm{n}=17\end{array}$ & 3.54 & 3.72 & 2.33 & 1.31 & $1.27-16.90$ & \multirow{2}{*}{0.024} \\
\hline & $\begin{array}{l}\text { Good } \\
\mathrm{n}=31\end{array}$ & 2.26 & 1.38 & 1.91 & 0.87 & $1.07-8.98$ & \\
\hline \multirow{2}{*}{ PIKЗСA } & $\begin{array}{c}\text { Poor } \\
\mathrm{n}=17\end{array}$ & 1.86 & 0.45 & 1.97 & 0.58 & $0.81-2.72$ & \multirow{2}{*}{0.483} \\
\hline & $\begin{array}{l}\text { Good } \\
\mathrm{n}=31\end{array}$ & 2.16 & 0.84 & 1.99 & 0.89 & $1.15-4.68$ & \\
\hline \multirow{2}{*}{ AKT3 } & $\begin{array}{c}\text { Poor } \\
\mathrm{n}=17\end{array}$ & 2.24 & 0.65 & 2.30 & 0.69 & $0.88-3.73$ & \multirow{2}{*}{0.822} \\
\hline & $\begin{array}{l}\text { Good } \\
\mathrm{n}=31\end{array}$ & 2.19 & 0.93 & 2.01 & 1.12 & $0.51-4.02$ & \\
\hline \multirow{2}{*}{ MDM2 } & $\begin{array}{c}\text { Poor } \\
\mathrm{n}=17\end{array}$ & 3.64 & 4.55 & 2.37 & 1.04 & $0.90-20.62$ & \multirow{2}{*}{0.296} \\
\hline & $\begin{array}{l}\text { Good } \\
\mathrm{n}=31\end{array}$ & 2.72 & 2.95 & 2.00 & 1.51 & $0.68-17.56$ & \\
\hline
\end{tabular}

Five-year survival was 87.02\% (95\% CI: 68.98-95.95); only four patients died at 50 to 65 months. Because of the low mortality, median survival could not be determined, and it was not possible to assess the relationship between each CNV and survival. 


\section{Discussion}

The neoadjuvant approach is a standard therapy option for operable early breast cancer, and has been associated with a very good $\mathrm{pCR}$ at the time of surgery. According to the results of Collaborative Trials in Neoadjuvant Breast Cancer (CTNeoBC) therapy in HER2-positive BC patients, women showing a pCR have a significantly better overall survival rate than those almost showing a pCR $[3,5]$. This has meant that a pathological complete response is regarded as an informative biomarker of survival to be considered in the approval of new treatments by the EMA (European Medicines Agency) [15]. Both the addition of $\mathrm{TZ}$ to neoadjuvant treatment in early $\mathrm{BC}$ patients and dual HER2 blockade with $\mathrm{TZ}+\mathrm{PZ}$ have led to significantly increased $\mathrm{pCR}$ rates [5,6]. In our study, BC patients showed a good response rate (Miller Payne grades $4-5$ ) both to TZ therapy and dual $\mathrm{TZ}+\mathrm{PZ}$ HER2 blockade (58.1\% and $76.5 \%$, respectively). Other authors have reported similar outcomes in larger patient series and concluded that neoadjuvant chemotherapy with $\mathrm{TZ}+\mathrm{PZ}$ increases the $\mathrm{pCR}$ rate compared with trastuzumab alone to one of around $50-70 \%$ [6]. However, it should be mentioned that approximately one-third of patients with HER2-positive early BC treated with TZ experience relapse [16]. This highlights a need to find good biomarkers of the efficacy of anti-HER2 treatment for this aggressive BC phenotype.

Mutations in genes with a role in the PI3K/AKT pathway have been examined in order to select patients who might benefit from anti-HER2 therapy, especially the dual blockade approach [1]. For instance, Han et al. suggested that HER2-positive early BC patients with a Val variant in the HER2Ile655Val SNP had a better survival when treated with TZ versus patients not receiving $\mathrm{TZ}$, although the typed mutation itself would confer a more aggressive phenotype [17]. Other studies have revealed that the presence of mutations in the PIK3 gene, seen in 12 to $39 \%$ of HER2-positive BC patients, can lead to resistance to trastuzumab therapy, showing a lower $\mathrm{pCR}$ rate and shorter disease-free survival [18]. The genes examined in our study-HER2, FGFR1, PIK3CA, AKT3 and MDM2-were selected on the grounds of their implication in this metabolic pathway and their known consequences on cell cycle regulation via inhibition of p53 [8]. Our results highlight that an amplified number of FGFR1 gene copies seems overall related to a significantly worse efficacy of treatment $(p=0.024$, Table 3$)$. Besides the whole patient sample, this poor response was also observed in the subgroup of patients treated only with TZ $(p=0.037)$ but not in the subgroup given the $\mathrm{TZ}+\mathrm{PZ}$ combo. The small sample size of both subgroups is a limitation of our study, preventing the observation of possible significant differences and multivariate analysis to check for interactions between $\mathrm{CNV}$ and other data such as histological stage, as performed by Fountzilas et al. with TP53 and PIK3CA mutation status [19]. Nevertheless, in women treated with $\mathrm{TZ}+\mathrm{PZ}$, having an amplified FGFR1 gene was effectively linked to a significantly lower $\mathrm{pCR}$ (Miller-Payne grade 5, $p=0.021$ ).

Amplification of the FGFR1 gene is its most common variation, occurring in $8-15 \%$ of all cases of breast cancer [20]. FGFR1 amplification has been considered a marker of resistance of cell lines to endocrine therapy in in vitro studies [21]. Our patients who were poor responders had both amplified FGFR1 and HER2 as all participants were HER2-positive at diagnosis (as an inclusion criterion). According to Chen et al., patients with FGFR1 and HER1/2 co-amplification exhibit a less favorable prognosis compared with patients with either FGFR1, HER1/2 amplification or with no amplification of these genes [14]. However, other studies have related FGFR1 amplification to an improved response of BC patients to other treatments. For instance, Hui et al. concluded that HR-/HER2-negative metastatic BC patients showed an apparent increase in overall response to lucitanib if they had a high number of FGFR1 copies [22]. Also seemingly relevant is the link detected between PI3K/AKT pathway activation and both worse trastuzumab efficacy and median time to progression and survival [23]. These last authors also reported an increased risk of disease progression in HER2-positive BC patients with mutations in the PIK3CA gene [23].

Several clinical trials have shown that $\mathrm{TZ}$ can significantly increase disease-free survival and overall survival in HER2-positive BC patients in both metastatic and early-stage 
settings (see for instance, [24]). The five-year survival rate in our study was $81.6 \%$, and only four patients died. Due to this low mortality, median survival could not be measured, and it was not possible to assess the link between each CNV and survival.

Hanker et al. described that the FGFR and HER2 pathways can cooperate to promote tumor progression as a result of somatic mutations in genes related to the former pathway that could play a role in resistance to anti-HER2 drugs in HER2-positive BC tumors [25]. These authors considered that patients showing FGFR1 expression in the bottom $80 \%$ percentile had a significantly reduced risk of disease recurrence when treated with $\mathrm{TZ}$. However, TZ did not reduce the risk of recurrence in the top 20\% FGFR1 expression percentile. Based on the results of in vitro and in vivo mice studies, Akhand et al. suggested that enhanced FGFR1 expression also confers resistance to anti-HER2 antibody therapies by disrupting trastuzumab's ability to bind to HER2 [26]. Although the mechanisms whereby FGFR prevents trastuzumab binding have not yet been determined, recent studies have unveiled the relevant role of micro-RNAs in gastric cancer HER2-positive cells. Guo et al. proposed that miR-301a-3p disseminated TZ resistance by activating the expression of FGFR1 through the intercellular transfer of this micro-RNA by exosomes [27].

The field of anti-cancer drug development is presently focused on the identification of biomarkers for more personalized treatment. In effect, CNVs have shown their potential utility for predicting the risk of disease progression in patients with solid tumors [28]. Our study in patients overexpressing the HER2 gene (HER2-positive) shows that FGFR1 gene amplification is associated with a poor tumor response to both anti-HER2 treatments, trastuzumab and trastuzumab plus pertuzumab. Although the sample size is a limitation, because of our results do not reach the minimum accepted $80 \%$ statistical power, the findings of this study provide direction for future studies designed to address this relationship. Future clinical trials should also explore whether resistance to anti-HER2 therapies could be diminished by HER2 and FGFR inhibitor drug combinations.

Author Contributions: A.F.-S. and D.M., conceived the project. A.F.-S. managed the funding support. D.M., B.A., and A.M. were responsible for data collection. M.G. headed the experiments. A.N. and A.R.-L. analyzed the data. A.F.-S. wrote manuscript. M.G., A.N. and A.R.-L. reviewed the manuscript. All authors have read and agreed to the published version of the manuscript.

Funding: This research was funded by Universidad Europea de Madrid (2019/UEM17) and by Foundation of the Universidad Europea, (grant numbers FGUE001804 and FGUE001805).

Institutional Review Board Statement: The study was conducted according to the guidelines of the Declaration of Helsinki and approved by the University Hospital of Fuenlabrada Ethics Committee (identification code: APR 18/20, September 2018).

Informed Consent Statement: Informed consent for the experimental use of biopsies/tumors collected for diagnosis was provided by all patients as study protocol required.

Data Availability Statement: All data available are reported in the article.

Acknowledgments: Thanks are expressed to Miguel Galán, Laura Beltrán and Cristina Andreu for many helpful discussion and statistical support.

Conflicts of Interest: The authors declare no conflict of interest. 


\section{References}

1. Loibl, S.; Gianni, L. HER2-positive breast cancer. Lancet 2017, 389, 2415-2429. [CrossRef]

2. Bray, F.; Ferlay, J.; Soerjomataram, I.; Siegel, R.L.; Torre, L.A.; Jemal, A. Global cancer statistics 2018: GLOBOCAN estimates of incidence and mortality worldwide for 36 cancers in 185 countries. CA Cancer J. Clin. 2018, 68, 394-424. Erratum in. CA Cancer J. Clin. 2020, 70, 313. [CrossRef]

3. Cortazar, P.; Zhang, L.; Untch, M. Pathological complete response and long-term clinical benefit in breast cancer: The CTNeoBC pooled analysis. Lancet 2014, 384, 164-172. [CrossRef]

4. Slamon, D.J.; Leyland-Jones, B.; Shak, S. Use of chemotherapy plus a monoclonal antibody against HER2 for metastatic breast cancer that overexpresses HER2. N. Engl. J. Med. 2001, 344, 783-792. [CrossRef]

5. Gianni, L.; Eiermann, W.; Semiglazov, V. Neoadjuvant chemotherapy with trastuzumab followed by adjuvant trastuzumab versus neoadjuvant chemotherapy alone in patients with HER2-positive locally advanced breast cancer (the NOAH trial): A randomised controlled superiority trial with a parallel HER2-negative cohort. Lancet 2010, 375, 377-384.

6. Takada, M.; Toi, M. Neoadjuvant treatment for HER2-positive breast cancer. Chin. Clin. Oncol. 2020, 9, 32. [CrossRef] [PubMed]

7. Turner, N.; Grose, R. Fibroblast growth factor signalling: From development to cancer. Nat. Rev. Cancer 2010, 10, 116-129. [CrossRef] [PubMed]

8. Abraham AGrr O'Neill, E. PI3K/Akt-mediated regulation of p53 in cancer. Biochem. Soc. Trans. 2014, 42, 798-803. [CrossRef] [PubMed]

9. Zhou, B.P.; Liao, Y.; Xia, W.; Zou, Y.; Spohn, B.; Hung, M.C. HER-2/neu induces p53 ubiquitination via Akt-mediated MDM2 phosphorylation. Nat. Cell Biol. 2001, 3, 973-982. Erratum in. Nat. Cell Biol. 2002, 4, 736. [CrossRef]

10. Andre, F.; Job, B.; Dessen, P.; Tordai, A.; Michiels, S.; Liedtke, C.; Richon, C.; Yan, K.; Wang, B.; Vassal, G.; et al. Molecular characterization of breast cancer with high-resolution oligonucleotide comparative genomic hybridization array. Clin. Cancer Res. 2009, 15, 441-451. [CrossRef]

11. Cancer Genome Atlas Network: Comprehensive molecular portraits of human breast tumours. Nature 2012, 490, 61-70. [CrossRef] [PubMed]

12. Slamon, D.; Eiermann, W.; Robert, N. Adjuvant trastuzumab in HER2-positive breast cancer. N. Engl. J. Med. 2011, 365, 1273-1283. [CrossRef] [PubMed]

13. Park, Y.H.; Shin, H.T.; Jung, H.H.; Choi, Y.L.; Ahn, T.; Park, K.; Lee, A.; Do, I.G.; Kim, J.Y.; Ahn, J.S.; et al. Role of HER2 mutations in refractory metastatic breast cancers: Targeted sequencing results in patients with refractory breast cancer. Oncotarget 2015, 6, 32027-32038. [CrossRef] [PubMed]

14. Chen, S.; Qiu, Y.; Guo, P.; Pu, T.; Feng, Y.; Bu, H. FGFR1 and HER1 or HER2 co-amplification in breast cancer indicate poor prognosis. Oncol. Lett. 2018, 15, 8206-8214. [CrossRef] [PubMed]

15. European Medicines Agency. EMA/CHMP/151853/2014: The Role of the Pathological Complete Response as an endpoint in Neoadjuvant Breast Cancer Studies. 20 March 2014. Available online: http://www.ema.europa.eu/docs/en_GB/document_ library/Scientific_guideline/2014/04/WC500165781.pdf (accessed on 8 April 2016).

16. Jagosky, M.; Tan, A.R. Combination of Pertuzumab and Trastuzumab in the Treatment of HER2-Positive Early Breast Cancer: A Review of the Emerging Clinical Data. Breast Cancer 2021, 13, 393-407. [CrossRef] [PubMed]

17. Han, X.; Diao, L.; Xu, Y.; Xue, W.; Ouyang, T.; Li, J.; Wang, T.; Fan, Z.; Fan, T.; Lin, B.; et al. Association between the HER2 Ile655Val polymorphism and response to trastuzumab in women with operable primary breast cancer. Ann. Oncol. 2014, 25, 1158-1164. [CrossRef]

18. Seo, Y.; Park, Y.H.; Ahn, J.S.; Im, Y.H.; Nam, S.J.; Cho, S.Y.; Cho, E.Y. PIK3CA Mutations and Neoadjuvant Therapy Outcome in Patients with Human Epidermal Growth Factor Receptor 2-Positive Breast Cancer: A Sequential Analysis. J. Breast Cancer 2018, 21, 382-390. [CrossRef]

19. Fountzilas, G.; Giannoulatou, E.; Alexopoulou, Z.; Zagouri, F.; Timotheadou, E.; Papadopoulou, K.; Lakis, S.; Bobos, M.; Poulios, C.; Sotiropoulou, M.; et al. TP53 mutations and protein immunopositivity may predict for poor outcome but also for trastuzumab benefit in patients with early breast cancer treated in the adjuvant setting. Oncotarget 2016, 7, 32731-32753. [CrossRef]

20. Elbauomy Elsheikh, S.; Green, A.R.; Lambros, M.B.; Turner, N.C.; Grainge, M.J.; Powe, D.; Ellis, I.O.; Reis-Filho, J.S. FGFR1 amplification in breast carcinomas: A chromogenic in situ hybridisation analysis. Breast Cancer Res. 2007, 9, R23. [CrossRef] [PubMed]

21. Turner, N.; Pearson, A.; Sharpe, R.; Lambros, M.; Geyer, F.; Lopez-Garcia, M.A.; Natrajan, R.; Marchio, C.; Iorns, E.; Mackay, A.; et al. FGFR1 amplification drives endocrine therapy resistance and is a therapeutic target in breast cancer. Cancer Res. 2010, 70, 2085-2094. [CrossRef] [PubMed]

22. Hui, R.; Pearson, A.; Cortes, J.; Campbell, C.; Poirot, C.; Azim, H.A., Jr.; Fumagalli, D.; Lambertini, M.; Daly, F.; Arahmani, A.; et al. Lucitanib for the Treatment of HR+/HER2- Metastatic Breast Cancer: Results from the Multicohort Phase II FINESSE Study. Clin. Cancer Res. 2020, 26, 354-363. [CrossRef] [PubMed]

23. Razis, E.; Bobos, M.; Kotoula, V.; Eleftheraki, A.G.; Kalofonos, H.P.; Pavlakis, K.; Papakostas, P.; Aravantinos, G.; Rigakos, G.; Efstratiou, I.; et al. Evaluation of the association of PIK3CA mutations and PTEN loss with efficacy of trastuzumab therapy in metastatic breast cancer. Breast Cancer Res. Treat. 2011, 128, 447-456. [CrossRef] 
24. Romond, E.H.; Perez, E.A.; Bryant, J.; Suman, V.J.; Geyer, C.E., Jr.; Davidson, N.E.; Tan-Chiu, E.; Silvana Martino, D.O.; Paik, S.; et al.; Kaufman, P.A.; et al. Trastuzumab plus adjuvant chemotherapy for operable HER2-positive breast cancer. N. Engl. J. Med. 2005, 353, 1673-1684. [CrossRef]

25. Hanker, A.B.; Garrett, J.T.; Estrada, M.V.; Moore, P.D.; Ericsson, P.G.; Koch, J.P.; Langley, E.; Singh, S.; Kim, P.S.; Frampton, G.M.; et al. HER2-Overexpressing Breast Cancers Amplify FGFR Signaling upon Acquisition of Resistance to Dual Therapeutic Blockade of HER2. Clin. Cancer Res. 2017, 23, 4323-4334. Erratum in. Clin. Cancer Res. 2019, 25, 1434. [CrossRef] [PubMed]

26. Akhand, S.S.; Chen, H.; Purdy, S.C.; Liu, Z.; Anderson, J.C.; Willey, C.D.; Wendt, M.K. Fibroblast growth factor receptor facilitates recurrence of minimal residual disease following trastuzumab emtansine therapy. NPJ Breast Cancer. 2021, 7, 5. [CrossRef] [PubMed]

27. Guo, J.; Zhong, X.; Tan, Q.; Yang, S.; Liao, J.; Zhuge, J.; Hong, Z.; Deng, Q.; Zuo, Q. miR-301a-3p induced by endoplasmic reticulum stress mediates the occurrence and transmission of trastuzumab resistance in HER2-positive gastric cancer. Cell Death Dis. 2021, 12, 696. [CrossRef] [PubMed]

28. Soong, D.; Stratford, J.; Avet-Loiseau, H.; Bahlis, N.; Davies, F.; Dispenzieri, A.; Sasser, A.K.; Schecter, J.M.; Qi, M.; Brown, C.; et al. CNV Radar: An improved method for somatic copy number alteration characterization in oncology. BMC Bioinform. 2020, 21, 98. [CrossRef] [PubMed] 